\title{
On a problem of Sárközy and Sós for multivariate linear forms
}

\author{
Juanjo Rué Christoph Spiegel*
}

\begin{abstract}
We prove that for pairwise co-prime numbers $k_{1}, \ldots, k_{d} \geq 2$ there does not exist any infinite set of positive integers $\mathcal{A}$ such that the representation function $r_{\mathcal{A}}(n)=\#\left\{\left(a_{1}, \ldots, a_{d}\right) \in\right.$ $\left.\mathcal{A}^{d}: k_{1} a_{1}+\cdots+k_{d} a_{d}=n\right\}$ becomes constant for $n$ large enough. This result is a particular case of our main theorem, which poses a further step towards answering a question of Sárközy and Sós and widely extends a previous result of Cilleruelo and Rué for bivariate linear forms (Bull. of the London Math. Society 2009).
\end{abstract}

\section{Javier Cilleruelo, in Memoriam}

\section{Introduction}

Let $\mathcal{A} \subseteq \mathbb{N}_{0}$ be an infinite set of positive integers and $k_{1}, \ldots, k_{d} \in \mathbb{N}$. We are interested in studying the behaviour of the representation function

$$
r_{\mathcal{A}}(n)=r_{\mathcal{A}}\left(n ; k_{1}, \ldots, k_{d}\right)=\#\left\{\left(a_{1}, \ldots, a_{d}\right) \in \mathcal{A}^{d}: k_{1} a_{1}+\cdots+k_{d} a_{d}=n\right\}
$$

More specifically, Sárközy and Sós [5, Problem 7.1.] asked for which values of $k_{1}, \ldots, k_{d}$ one can find an infinite set $\mathcal{A}$ such that the function $r_{\mathcal{A}}\left(n ; k_{1}, \ldots, k_{d}\right)$ becomes constant for $n$ large enough. For the base case, it is clear that $r_{\mathcal{A}}(n ; 1,1)$ is odd whenever $n=2 a$ for some $a \in \mathcal{A}$ and even otherwise, so that the representation function cannot become constant. For $k \geq 2$, Moser [3] constructed a set $\mathcal{A}$ such that $r_{\mathcal{A}}(n ; 1, k)=1$ for all $n \in \mathbb{N}_{0}$. The study of bivariate linear forms was completely settled by Cilleruelo and the first author [1] by showing that the only cases in which $r_{\mathcal{A}}\left(n ; k_{1}, k_{2}\right)$ may become constant are those considered by Moser.

The multivariate case is less well studied. If $\operatorname{gcd}\left(k_{1}, \ldots, k_{d}\right)>1$, then one trivially observes that $r\left(n ; k_{1}, \ldots, k_{d}\right)$ cannot become constant. The only non-trivial case studied so far was the following: for $m>1$ dividing $d$, the first author [4] showed that if in the $d$-tuple of coefficients

${ }^{*}$ Universitat Politècnica de Catalunya, Department of Mathematics, Edificio Omega, 08034 Barcelona, Spain, and Barcelona Graduate School of Mathematics. E-mail: juan.jose.rue@upc.edu, christoph.spiegel@upc.edu. Supported by the Spanish Ministerio de Economía y Competitividad projects MTM2014-54745-P, MTM2017-82166-P and the María de Maetzu research grant MDM-2014-0445. C. S. is also suppported by an FPI grant under the project projects MTM2014-54745-P. 
$\left(k_{1}, \ldots, k_{d}\right)$ each element is repeated $m$ times, then there cannot exists an infinite set $\mathcal{A}$ such that $r_{\mathcal{A}}\left(n ; k_{1}, \ldots, k_{d}\right)$ becomes constant for $n$ large enough. This for example covers the case $\left(k_{1}, k_{2}, k_{3}, k_{4}, k_{5}, k_{6}\right)=(2,4,6,2,4,6)$. Observe that each coefficient in this example is repeated twice, that is $m=2$.

In this paper we provide a step beyond this result and show that whenever the set of coefficients is pairwise co-prime, then there does not exists any infinite set $\mathcal{A}$ for which $r\left(n ; k_{1}, \ldots, k_{d}\right)$ is constant for $n$ large enough. This is a particular case of our main theorem, which covers a wide extension of this situation:

Theorem 1.1. Let $q_{1}, \ldots, q_{m} \geq 2$ be pairwise co-prime integers and $b(i, j) \in\{0,1\}$, so that for each $1 \leq i \leq d$ there exists some $1 \leq j \leq m$ such that $b(i, j)=1$. If $k_{i}=q_{1}^{b(i, 1)} \cdots q_{m}^{b(i, m)}$ for $1 \leq i \leq d$, then for every infinite set $\mathcal{A} \subseteq \mathbb{N}_{0}$ the function $r_{\mathcal{A}}\left(n ; k_{1}, \ldots, k_{d}\right)$ cannot become constant.

In particular, if $m=d$ and $b(i, j)=1$ if and only if $i=j$, then this represents the case where $k_{1}, \ldots, k_{d} \geq 2$ are pairwise co-prime numbers. Other new cases covered by this result are for instance $\left(k_{1}, k_{2}, k_{3}\right)=(2,3,2 \times 3)$ as well as $\left(k_{1}, k_{2}, k_{3}, k_{4}\right)=\left(2^{2} \times 3,2^{2} \times 5,3 \times 5,2^{2} \times 3 \times 5\right)$.

Our method starts with some ideas introduced in [1] dealing with generating functions and cyclotomic polyomials (see Section 2). The main new idea in this paper is to use an inductive argument in order to be able to show that a certain multivariate recurrence relation is not possible to be satisfied unless some initial condition is trivial.

\section{Preliminaries}

Generating functions. The language in which we will approach this problem goes back to [2]. Let $f_{\mathcal{A}}(z)=\sum_{a \in \mathcal{A}} z^{a}$ denote the generating function associated with $\mathcal{A}$. By a simple argument over the generating functions, it is easy to verify that the existence of a set $\mathcal{A}$ for which $r_{\mathcal{A}}\left(n ; k_{1}, \ldots, k_{d}\right)$ becomes constant would imply that

$$
f_{\mathcal{A}}\left(z^{k_{1}}\right) \cdots f_{\mathcal{A}}\left(z^{k_{d}}\right)=\frac{P(z)}{1-z}
$$

for some polynomial $P$ with positive integer coefficients satisfying $P(1) \neq 0$. To simplify notation, we will generally consider the $d$-th power of this equation, that is for $F_{\mathcal{A}}(z)=f_{\mathcal{A}}^{d}(z)$ we have

$$
F_{\mathcal{A}}\left(z^{k_{1}}\right) \cdots F_{\mathcal{A}}\left(z^{k_{d}}\right)=\frac{P^{d}(z)}{(1-z)^{d}}
$$

Let us remark two obvious but important properties of $f_{\mathcal{A}}$ and $F_{\mathcal{A}}$.

Remark 2.1. $f_{\mathcal{A}}(z)$ is a formal power series with coefficients in $\{0,1\}$ that therefore is analytic in the open complex disc $\mathcal{D}=\{z \in \mathbb{C}:|z|<1\}$. It follows that $F_{\mathcal{A}}(z)$ is also a formal power series with positive coefficients that is analytic in $\mathcal{D}$. 
This is the starting point of the proof of Theorem 1.1, mainly building upon the tools developed in [1] using properties relating to cyclotomic polynomials that we will now briefly review.

Cyclotomic polynomials. The cyclotomic polynomial of order $n$ is defined as

$$
\Phi_{n}(z)=\prod_{\xi \in \phi_{n}}(z-\xi) \in \mathbb{Z}[z]
$$

where

$$
\phi_{n}=\left\{e^{\frac{2 \pi i \ell}{n}}: 0 \leq \ell<n \text { satisfying }(\ell, n)=1\right\}=\left\{\xi \in \mathbb{C}: \xi^{k}=1 \text { iff } k \equiv 0 \bmod n\right\}
$$

denotes the set of primitive roots of unity of order $n \in \mathbb{N}$. It is well known that $\Phi_{n}(z) \in \mathbb{Z}[z]$, that is it has integer coefficients. Cyclotomic polynomials have the property of being irreducible over $\mathbb{Z}[z]$ and therefore it follows that for any polynomial $P(z) \in \mathbb{Z}[z]$ and $n \in \mathbb{N}$ there exists an integer $s_{n} \in \mathbb{N}_{0}$ such that

$$
P_{n}(z):=P(z) \Phi_{n}^{-s_{n}}(z)
$$

is a polynomial in $\mathbb{Z}[z]$ satisfying $P_{n}(\xi) \neq 0$ for all $\xi \in \phi_{n}$. We will say that we have factored $\Phi_{n}(z)$ out of $P(z)$ with multiplicity $s_{n}$. Note that the multiplicity is trivially unique.

This is not guaranteed to be possible for arbitrary non-polynomial functions. In particular, our function $F_{\mathcal{A}}(z)$ is not even analytic at roots of unity and it can also be shown that even the radial limit of $F_{\mathcal{A}}(z)$, where $z$ approaches some root of unit $\xi$ radially from within $\mathcal{D}$, may not exist in general. However, we can extend our notion of factoring out cyclotomic polynomials in a natural way that will be applicable to our function $F_{\mathcal{A}}(z)$.

Definition 2.2. Let $n \in \mathbb{N}$ and $F(z)$ some function analytic in $\mathcal{D}$. We say that we can factor $\Phi_{n}(z)$ out of $F(z)$ with multiplicity $r_{n}$ if, for any $\xi \in \phi_{n}$ and sequence $\left\{z_{k}: k \in \mathbb{N}\right\} \subset[0,1)$ converging to 1 , the limit of $\left|F\left(z_{k} \xi\right) \Phi_{n}^{-r_{n}}\left(z_{k} \xi\right)\right|$ as $k \rightarrow \infty$ either does not exist or is not equal to 0 or $\infty$.

Note that $\left|F\left(z_{k} \xi\right) \Phi_{n}^{-r_{n}}\left(z_{k} \xi\right)\right|$ not going to infinity is the same as $F\left(z_{k} \xi\right) \Phi_{n}^{-r_{n}}\left(z_{k} \xi\right)$ being bounded. We remark that, by continuity, this notion is a true extension of the previous one for polynomials. It is also again easy to verify that the multiplicity, if it exists, is uniquely determined.

Lemma 2.3. If we can factor $\Phi_{n}(z)$ out of $F(z)$, then the multiplicity is uniquely determined. Proof. Assume that we can factor $\Phi_{n}(z)$ out of $F(z)$ with multiplicity $r_{n}$. Let $\left\{z_{k}: k \in \mathbb{N}\right\} \subset$ $[0,1)$ be a sequence converging to 1 and $\xi \in \phi_{n}$. Consider

$$
\left|F\left(z_{k} \xi\right) \Phi_{n}^{-r_{n}+\alpha}\left(z_{k} \xi\right)\right|=\left|F\left(z_{k} \xi\right) \Phi_{n}^{-r_{n}}\left(z_{k} \xi\right)\right|\left|\Phi_{n}^{\alpha}\left(z_{k} \xi\right)\right|
$$


as $k$ goes to infinity. As $\Phi_{n}(\xi)=0$ and $F\left|\left(z_{k} \xi\right) \Phi_{n}^{-r_{n}}\left(z_{k} \xi\right)\right|$ is bounded and does not go to 0 , (44) must tend to 0 if $\alpha>0$ and to $\infty$ if $\alpha<0$. It follows that the multiplicity must be uniquely determined.

Let us introduce some short-hand notation for this that we will use in the next section. If $q_{1}, \ldots, q_{m}$ are fixed co-prime integers as given by Theorem 1.1 and $\mathbf{j}=\left(j_{1}, \ldots, j_{m}\right) \in \mathbb{N}_{0}^{m}$, then we write

$$
\Phi_{\mathbf{j}}(z):=\Phi_{q_{1}^{j_{1} \ldots q_{m}^{j_{m}}}}(z), \quad \phi_{\mathbf{j}}:=\phi_{q_{1}^{j_{1} \ldots q_{m}^{j_{m}}}}, \quad s_{\mathbf{j}}:=s_{q_{1}^{j_{1} \ldots} q_{m}^{j_{m}}} \text { and } r_{\mathbf{j}}:=r_{q_{1}^{j_{1} \ldots} q_{m}^{j_{m}}} .
$$

The main strategy of the proof is to show that for any $\mathbf{j} \in \mathbb{N}_{0}^{m}$ we can factor $\Phi_{\mathbf{j}}(z)$ out of our hypothetical function $F_{\mathcal{A}}(z)=f_{\mathcal{A}}^{d}(z)$ satisfying (11) and that the multiplicites $r_{\mathbf{j}}$ have to fulfil certain relations between themselves. The goal will be to find a contradiction in these relations, negating the possibility of such a function and therefore such a set $\mathcal{A}$ existing in the first place. Before formally establishing these relations in the next section, let us introduce two lemmata that we will need.

Lemma 2.4. Given $k, n \in \mathbb{N}$ such that $k \mid n$ we have $\phi_{n / k}=\left\{\xi^{k}: \xi \in \phi_{n}\right\}$. Furthermore, we can factor $\Phi_{n}(z)$ out of $\Phi_{n / k}\left(z^{k}\right)$ with multiplicity 1 .

Proof. To see equality between the two sets, observe that

$$
\begin{aligned}
\left\{\xi^{k}: \xi \in \phi_{n}\right\} & =\left\{\xi^{k}: \xi^{\ell}=1 \text { iff } \ell \equiv 0 \bmod n\right\} \\
& =\left\{\xi^{k}:\left(\xi^{k}\right)^{\ell / k}=1 \text { iff } \ell \equiv 0 \bmod n\right\} \\
& =\left\{\xi^{k}:\left(\xi^{k}\right)^{\ell}=1 \text { iff } \ell \equiv 0 \bmod n / k\right\}=\phi_{n / k} .
\end{aligned}
$$

As $\Phi_{n / k}\left(z^{k}\right)$ is a polynomial in $\mathbb{Z}[z]$ and $\Phi_{n / k}\left(\xi^{k}\right)=0$ for any $\xi \in \phi_{n}$ via the previous observation, it follows that we can factor out $\Phi_{n}(z)$. The multiplicity is equal to 1 since all roots of $\Phi_{n / k}\left(z^{k}\right)$ are simple.

Lastly, we will also need the following technical lemma that will allow us to draw conclusions from the limit of certain types of products to the limits of its individual factors.

Lemma 2.5. Let $F(z)=\sum_{n \in \mathbb{N}} a_{n} z^{n}$ be a formal power series with positive coefficients that is analytic in $\mathcal{D}$. If there exists a sequence $\left\{z_{k}: k \in \mathbb{N}\right\} \subset[0,1)$ tending to 1 such that $\left|F\left(z_{k}\right)\left(1-z_{k}\right)\right|$ goes to 0 , then so does $\left|F\left(z_{k}^{\alpha}\right)\left(1-z_{k}^{\alpha}\right)\right|$ for any $\alpha \neq 0$. If $\left|F\left(z_{k}\right)\left(1-z_{k}\right)\right|$ goes to $\infty$, then so does $\left|F\left(y_{k}^{\alpha}\right)\left(1-y_{k}^{\alpha}\right)\right|$ for some subsequence $\left\{y_{k}: k \in \mathbb{N}\right\} \subseteq\left\{z_{k}: k \in \mathbb{N}\right\}$ also tending to 1 .

Proof. We start by observing that, as $F(z)$ has positive coefficients, we may omit the absolute values since $|F(z)(1-z)|=F(z)(1-z)$ for any $0 \leq z<1$. Let us start with the first case, that is $F\left(z_{k}\right)\left(1-z_{k}\right)$ going to 0 . We have

$$
\left|F\left(z_{k}\right)\left(1-z_{k}\right)-F\left(z_{k}^{\alpha}\right)\left(1-z_{k}^{\alpha}\right)\right|=\left|\sum_{n} a_{n} z_{k}^{n}\left(1-z_{k}\right)-\sum_{n} a_{n} z_{k}^{\alpha n}\left(1-z_{k}^{\alpha}\right)\right|
$$




$$
\begin{aligned}
& =\left|\sum_{n} a_{n} z_{k}^{n}\left(\left(1-z_{k}\right)-z_{k}^{(\alpha-1) n}\left(1-z_{k}^{\alpha}\right)\right)\right| \\
& \leq \sum_{n}\left|a_{n} z_{k}^{n}\right|\left|\left(1-z_{k}\right)-z_{k}^{(\alpha-1) n}\left(1-z_{k}^{\alpha}\right)\right| \\
& \leq \sum_{n}\left|a_{n} z_{k}^{n}\right|=F\left(z_{k}\right) \rightarrow 0 .
\end{aligned}
$$

In the last equality we have used the fact that the coefficients $a_{n}$ are positive and that $z_{k} \in[0,1)$ so that $\left|a_{n} z_{k}^{n}\right|=a_{n} z_{k}^{n}$. It clearly follows that $F\left(z_{k}^{\alpha}\right)\left(1-z_{k}^{\alpha}\right)$ must go to 0 as well.

Next, assume that $F\left(z_{k}\right)\left(1-z_{k}\right)$ goes to $\infty$ but that $F\left(z_{k}^{\alpha}\right)\left(1-z_{k}^{\alpha}\right)$ is bounded, that is $F\left(z_{k}^{\alpha}\right)\left(1-z_{k}^{\alpha}\right) \in[-M, M]$ for some $M \in \mathbb{R}$ and any $k \in \mathbb{N}$. It follows that

$$
\begin{aligned}
\left|F\left(z_{k}\right)\left(1-z_{k}\right)-F\left(z_{k}^{\alpha}\right)\left(1-z_{k}^{\alpha}\right)\right| & =\left|\sum_{n} a_{n} z_{k}^{\alpha n}\left(z_{k}^{(1-\alpha) n}\left(1-z_{k}\right)-\left(1-z_{k}^{\alpha}\right)\right)\right| \\
& \leq \sum_{n}\left|a_{n} z_{k}^{\alpha n}\right|\left|z_{k}^{(1-\alpha) n}\left(1-z_{k}\right)-\left(1-z_{k}^{\alpha}\right)\right| \\
& \leq \sum_{n}\left|a_{n} z_{k}^{\alpha n}\right|=F\left(z_{k}^{\alpha}\right) \leq M
\end{aligned}
$$

a contradiction since $\left|F\left(z_{k}\right)\left(1-z_{k}\right)-F\left(z_{k}^{\alpha}\right)\left(1-z_{k}^{\alpha}\right)\right| \rightarrow \infty$. Since $F\left(z_{k}^{\alpha}\right)\left(1-z_{k}^{\alpha}\right)$ must therefore be unbounded, there exists some subsequence $\left\{y_{k}: k \in \mathbb{N}\right\} \subset\left\{z_{k}: k \in \mathbb{N}\right\} \subset[0,1)$ such that $F\left(y_{k}^{\alpha}\right)\left(1-y_{k}^{\alpha}\right)$ goes to $\infty$.

\section{Recurrence relations}

We can now give the statement and proof establishing that we can factor any $\Phi_{\mathbf{j}}(z)$ out of our function $F_{\mathcal{A}}(z)$ and that the multiplicities satisfy certain relations. We will in fact state this for any $k_{1}, \ldots, k_{d} \in \mathbb{N}$ and later derive a contradiction from these relations in the specific case stated in Theorem 1.1

For any $a, b \in \mathbb{N}_{0}, \mathbf{j}=\left(j_{1}, \ldots, j_{m}\right) \in \mathbb{N}_{0}^{m}$ and $\mathbf{b}=\left(b_{1}, \ldots, b_{m}\right) \in \mathbb{N}_{0}^{m}$, we will use the notation

$$
a \ominus b=\max \{a-b, 0\} \quad \text { and } \quad \mathbf{j} \ominus \mathbf{b}=\left(j_{1} \ominus b_{1}, \ldots, j_{m} \ominus b_{m}\right) .
$$

Let us state the central proposition of this section.

Proposition 3.1. Let $q_{1}, \ldots, q_{m} \geq 2$ be pairwise co-prime integers and $k_{i}=q_{1}^{b(i, 1)} \cdots q_{m}^{b(i, m)}$ for $1 \leq i \leq d$ where $b(i, j) \in \mathbb{N}_{0}$. Furthermore, let $P(z) \in \mathbb{Z}[z]$ be a polynomial satisfying $P(1) \neq 0$ and $F(z)$ a formal power series with positive coefficients that is analytic in $\mathcal{D}$ such that

$$
F\left(z^{k_{1}}\right) \cdots F\left(z^{k_{d}}\right)=\frac{P^{d}(z)}{(1-z)^{d}} .
$$

Then for all $\boldsymbol{j} \in \mathbb{N}_{0}^{m}$ there exist integers $r_{\boldsymbol{j}} \in \mathbb{N}_{0}$ so that we can factor $\Phi_{j}$ out of $F$ with multiplicity $r_{j}$. Writing $\boldsymbol{b}_{i}=(b(i, 1), \ldots, b(i, m))$ for $1 \leq i \leq m$ as well as $s_{j} \in \mathbb{N}_{0}$ for the integer satisfying $P(\xi) \Phi_{j}^{-s_{j}}(\xi) \neq 0$ for any $\xi \in \phi_{j}$, these multiplicities satisfy the relations

$$
r_{\mathbf{0}}=-1 \quad \text { and } \quad r_{\boldsymbol{j} \ominus \boldsymbol{b}_{1}}+\cdots+r_{\boldsymbol{j} \ominus \boldsymbol{b}_{d}}=d s_{\boldsymbol{j}} \quad \text { for all } \boldsymbol{j} \in \mathbb{N}_{0}^{m} \backslash\{\mathbf{0}\}
$$


and we have $r_{i} \equiv-1 \bmod d$ for all $\boldsymbol{i} \in \mathbb{N}_{0}^{m}$.

Proof. We start assuming that the set of multiplicities $\left\{r_{\mathbf{j}}: \mathbf{j} \in \mathbb{N}_{0}^{m}\right\}$ exists and show that the relations given by (6) must be satisfied. After this, we will show that there is a way to recursively determine the values $\left\{r_{\mathbf{j}}: \mathbf{j} \in \mathbb{N}_{0}^{m}\right\}$, proving their existence.

Let us start with $r_{\mathbf{0}}=-1$. For $F_{\mathbf{0}}(z):=F(z)(1-z)$ we wish to show that there does not exist any sequence $\left\{z_{k}: k \in \mathbb{N}\right\} \subset[0,1)$ going to 1 such that $\left|F_{\mathbf{0}}\left(z_{k}\right)\right|$ tends to either 0 or $\infty$. Note that if such a sequence were to exist, then by iteratively applying Lemma 2.5 we would obtain some subsequence $\left\{y_{k}: k \in \mathbb{N}\right\} \subseteq\left\{z_{k}: k \in \mathbb{N}\right\}$ still tending to 1 such that all $\left|F_{\mathbf{0}}\left(y_{k}^{k_{\ell}}\right)\right|$ would collectively tend to either 0 or $\infty$ for any $1 \leq \ell \leq d$.

Inserting the equality $F(z)=(1-z)^{-1} F_{\mathbf{0}}(z)$ into (5) and observing that $(1-z) /\left(1-z^{k_{\ell}}\right)=$ $\left(1+z+\cdots+z^{k_{\ell}-1}\right)^{-1}$, we get that $F_{\mathbf{0}}(z)$ satisfies

$$
\prod_{\ell=1}^{d}\left|\left(1+z+\cdots+z^{k_{\ell}-1}\right)^{-1}\right|\left|F_{0}\left(z^{k_{\ell}}\right)\right|=\left|P^{d}(z)\right| .
$$

As $P^{d}(1) \neq 0$ as well as $\left(1+1+\cdots+1^{k_{\ell}-1}\right)^{-1}=1 / k_{\ell} \neq 0$ for $1 \leq \ell \leq d$ it follows that there cannot exist a sequence $\left\{z_{k}: k \in \mathbb{N}\right\} \subset[0,1)$ tending to 1 such that $\left|F_{\mathbf{0}}\left(z^{k_{\ell}}\right)\right|$ all collectively tend to 0 or $\infty$ for any $1 \leq \ell \leq d$, proving the desired statement.

Next, let us show that if for a given $\mathbf{j} \in \mathbb{N}_{0}^{m} \backslash\{\mathbf{0}\}$ the values $r_{\mathbf{j} \ominus \mathbf{b}_{1}}, \ldots, r_{\mathbf{j} \ominus \mathbf{b}_{d}}$ exist, then they must satisfy the relation given by ([6). For $1 \leq i \leq d$ let

$$
F_{\mathbf{j} \ominus \mathbf{b}_{i}}:=F(z) \Phi_{\mathbf{j} \ominus \mathbf{b}_{i}}^{-r_{\mathbf{j}} \mathbf{b}_{i}}
$$

and rewrite (5) as

$$
\Phi_{\mathbf{j} \ominus \mathbf{b}_{1}}^{r_{\mathbf{j} \ominus \mathbf{b}_{1}}}\left(z^{k_{1}}\right) F_{\mathbf{j} \ominus \mathbf{b}_{1}}\left(z^{k_{1}}\right) \cdots \Phi_{\mathbf{j} \ominus \mathbf{b}_{d}}^{r_{\mathbf{j} \ominus \mathbf{b}_{d}}}\left(z^{k_{d}}\right) F_{\mathbf{j} \ominus \mathbf{b}_{d}}\left(z^{k_{d}}\right)=\frac{\Phi_{\mathbf{j}}^{d s_{\mathbf{j}}}(z) P_{\mathbf{j}}^{d}(z)}{(1-z)^{d}} .
$$

Writing $R_{\mathbf{j}, i}(z):=\Phi_{\mathbf{j} \ominus \mathbf{b}_{i}}\left(z^{k_{i}}\right) \Phi_{\mathbf{j}}^{-1}(z)$ we can restate (7) as

$$
\Phi_{\mathbf{j}}^{r_{\mathbf{j} \ominus \mathbf{b}_{1}}+\cdots+r_{\mathbf{j} \ominus \mathbf{b}_{d}}-d s_{\mathbf{j}}}(z)\left(R_{\mathbf{j}, 1}^{r_{\mathbf{j} \ominus \mathbf{b}_{1}}}(z) F_{\mathbf{j} \ominus \mathbf{b}_{1}}\left(z^{k_{1}}\right) \cdots R_{\mathbf{j}, d}^{r_{\mathbf{j} \ominus \mathbf{b}_{d}}}(z) F_{\mathbf{j} \ominus \mathbf{b}_{d}}\left(z^{k_{d}}\right)\right)=\frac{P_{\mathbf{j}}^{d}(z)}{(1-z)^{d}} .
$$

We observe that, by assumption as well as Lemma 2.4, if we substitute $z_{k} \xi$ into (8) where $\xi \in \phi_{\mathbf{j}}$ and $\left\{z_{k}: k \in \mathbb{N}\right\} \subset[0,1)$ tends to 1 and take absolute values, then all involved factors but the first one converge neither to 0 nor to $\pm \infty$. As $\Phi_{\mathbf{j}}^{\alpha}\left(z_{k} \xi\right)$ tends to either 0 or $\infty$ for any $\alpha \neq 0$, it follows that the desired relation must hold.

It remains to be shown that the values $r_{\mathbf{j}}$ actually exist for any $\mathbf{j} \in \mathbb{N}_{0}$. We will do so recursively with the base case of $r_{\mathbf{0}}=-1$ already having been established. From now on, let us - for simplicities sake - redefine the value $s_{\mathbf{0}}$ (which previously was 0 as $P(0) \neq 0$ ) to be $s_{0}=-1$, so that the initial relation $r_{\mathbf{0}}=-1$ is now included in the general relation for the case 
$\mathbf{j}=\mathbf{0}$. We observe that if for some $1 \leq \ell \leq d$ all values $r_{\mathbf{j} \ominus \mathbf{b}_{1}}, \ldots, r_{\mathbf{j} \ominus \mathbf{b}_{d}}$ except for $r_{\mathbf{j} \ominus \mathbf{b}_{\ell}}$ have already been shown to exist, then through the already established (8) it is clear that setting

$$
r_{\mathbf{j} \ominus \mathbf{b}_{\ell}}=d s_{\mathbf{j}}-\sum_{i \neq \ell} r_{\mathbf{j} \ominus \mathbf{b}_{i}}
$$

would give the desired property, that is for no sequence $\left\{z_{k}: k \in \mathbb{N}\right\} \subset[0,1)$ going to 1 and $\xi \in \phi_{\mathbf{j} \ominus \mathbf{b}_{\ell}}$ could $F\left(z_{k} \xi\right) \Phi_{\mathbf{j} \ominus \mathbf{b}_{\ell}}^{-r_{\mathbf{j} \ominus \mathbf{b}_{\ell}}}\left(z_{k} \xi\right)$ go to either 0 or $\pm \infty$. We therefore wish to show inductively that for all $\mathbf{i} \in \mathbb{N}_{0}^{m}$ there exists a $\mathbf{j} \in \mathbb{N}_{0}^{m}$ and $1 \leq \ell \leq d$ such that $\mathbf{i}=\mathbf{j} \ominus \mathbf{b}_{\ell}$ and all other involved values $\mathbf{j} \ominus \mathbf{b}_{1}, \ldots, \mathbf{j} \ominus \mathbf{b}_{\ell-1}, \mathbf{j} \ominus \mathbf{b}_{\ell+1}, \ldots, \mathbf{j} \ominus \mathbf{b}_{d}$ have already been determined by the inductive hypothesis.

For this we will give the indices $\mathbf{j} \in \mathbb{N}_{0}^{m}$ inducing these relations an appropriate ordering. More preciesly, for each $\mathbf{j}=\left(j_{1}, \ldots, j_{m}\right) \in \mathbb{N}_{0}^{m}$ let $\mathbf{j}^{\leq}=\left(j_{1}^{\leq}, \ldots, j_{m}^{\leq}\right)$denote the ordered version, that is $j_{1}^{\leq} \leq j_{2}^{\leq} \leq \cdots \leq j_{m}^{\leq}$and there exists some permutation $\sigma$ on $m$ letters such that $\mathbf{j}=\left(j_{\sigma(1)}^{\leq}, \ldots, j_{\sigma(m)}^{\leq}\right)$. Consider the ordering on $\mathbb{N}_{0}^{m}$ given by $\mathbf{j} \prec \mathbf{j}^{\prime}$ if $\mathbf{j}^{\leq}$lexicographically comes before $\mathbf{j}^{\prime<}$. In this situation, ties are broken arbitrarily. We want to show that going through the indices $\mathbf{j}$ in that order and considering the relation $r_{\mathbf{j} \ominus \mathbf{b}_{1}}+\cdots+r_{\mathbf{j} \ominus \mathbf{b}_{d}}=d s_{\mathbf{j}}$, then at most one of the $r_{\mathbf{j} \ominus \mathbf{b}_{\ell}}$ will not have occurred in any of the previous relations given by some $\mathbf{j}^{\prime} \prec \mathbf{j}$.

Assume to the contrary that there exist $\mathbf{i} \neq \mathbf{i}^{\prime} \in \mathbb{N}_{0}^{m}$ such that, for both of them, $\mathbf{j} \in \mathbb{N}_{0}^{m}$ is the first index for which there exist $1 \leq \ell, \ell^{\prime} \leq d$ satisfying $\mathbf{i}=\mathbf{j} \ominus \mathbf{b}_{\ell}$ and $\mathbf{i}^{\prime}=\mathbf{j} \ominus \mathbf{b}_{\ell^{\prime}}$. Note that $\mathbf{b}_{\ell} \neq \mathbf{b}_{\ell^{\prime}}$ and therefore at least one of the two statements $\mathbf{j} \ominus\left(\mathbf{b}_{\ell}-\mathbf{b}_{\ell^{\prime}}\right) \prec \mathbf{j}$ and $\mathbf{j} \ominus\left(\mathbf{b}_{\ell^{\prime}}-\mathbf{b}_{\ell}\right) \prec \mathbf{j}$ must hold. To see this, assume without loss of generality that $\mathbf{j}=\left(j_{1}, \ldots, j_{m}\right)$ is already in ordered form. Note that $\mathbf{b}_{\ell}-\mathbf{b}_{\ell^{\prime}} \neq \mathbf{0}$ as $\mathbf{i} \neq \mathbf{i}^{\prime}$. Writing $\mathbf{b}_{\ell}=\left(b_{1}, \ldots, b_{m}\right)$ and $\mathbf{b}_{\ell^{\prime}}=\left(b_{1}^{\prime}, \ldots, b_{m}^{\prime}\right)$, and letting $1 \leq i \leq m$ be the first index such that $b_{i} \neq b_{i}^{\prime}$ and $j_{i}>0$, then we clearly have that either

$$
j_{i} \ominus\left(b_{i}-b_{i^{\prime}}\right)=\max \left\{j_{i}-\left(b_{i}-b_{i^{\prime}}\right), 0\right\}<j_{i} \quad \text { or } \quad j_{i} \ominus\left(b_{i^{\prime}}-b_{i}\right)=\max \left\{j_{i}+\left(b_{i}-b_{i^{\prime}}\right), 0\right\}<j_{i}
$$

meaning that at least one of the two values $\mathbf{j} \ominus\left(\mathbf{b}_{\ell}-\mathbf{b}_{\ell^{\prime}}\right)$ and $\mathbf{j} \ominus\left(\mathbf{b}_{\ell^{\prime}}-\mathbf{b}_{\ell}\right)$ must lexicographically come before $\mathbf{j}$. Note that such index $i$ must exist since if $j_{i}=0$ whenever $b_{i}-b_{i}^{\prime} \neq 0$ then we would have had $\mathbf{i}=\mathbf{j} \ominus \mathbf{b}_{\ell}=\mathbf{b}_{\ell^{\prime}}=\mathbf{i}^{\prime}$ in contradiction to our assumption that $\mathbf{i} \neq \mathbf{i}^{\prime}$.

Assume now without loss of generality that $\mathbf{j} \ominus\left(\mathbf{b}_{\ell}-\mathbf{b}_{\ell^{\prime}}\right) \prec \mathbf{j}$. Since for $a, b, c \geq 0$ we trivially have that $\max \{\max \{a-b+c, 0\}-c, 0\}=\max \{\max \{a-b,-c\}, 0\}=\max \{a-b, 0\}$, it follows that

$$
\left(\mathbf{j} \ominus\left(\mathbf{b}_{\ell}-\mathbf{b}_{\ell^{\prime}}\right)\right) \ominus \mathbf{b}_{\ell^{\prime}}=\mathbf{j} \ominus \mathbf{b}_{\ell}=\mathbf{i} .
$$

This is however in contradiction to the requirement that $\mathbf{j}$ was the smallest index with respect to the ordering $\prec$ for which the relation given by (6) involves $r_{\mathbf{i}}$, giving us the desired result.

Finally, note that from the previous argument it also inductively follows that $r_{\mathbf{i}} \equiv-1 \bmod d$ for all $\mathbf{i} \in \mathbb{N}_{0}^{m}$ as in the base case we have that $r_{\mathbf{0}}=-1$. 


\section{Proof of Theorem 1.1}

We will now use the proposition established in the previous section to prove Theorem 1.1 by contradiction. We start by introducing some necessary notation and definitions. We write $\mathbf{c}_{i}=(c(i, 1), \ldots, c(i, m)) \in \mathbb{N}_{0}^{m}$ and for any $1 \leq \ell \leq m$ we use the notation

$$
S_{\ell}=\{1 \leq i \leq d: c(i, \ell)=0\} \quad \text { and } \quad S_{\ell}^{\prime}=\{1, \ldots, d\} \backslash S_{\ell}
$$

We will also use the following notation: for any $\mathbf{i}=\left(i_{1}, \ldots, i_{m-1}\right) \in \mathbb{N}_{0}^{m-1}$ and $1 \leq \ell \leq m$ let

$$
\Delta_{\mathbf{i}, \ell}=v_{\left(i_{1}, \ldots, i_{\ell-1}, 1, i_{\ell}, \ldots, i_{m-1}\right)}-v_{\left(i_{1}, \ldots, i_{\ell-1}, 0, i_{\ell}, \ldots, i_{m-1}\right)} .
$$

Finally, for $1 \leq \ell \leq m$, we write $\mathbb{1}_{\ell} \in \mathbb{N}_{0}^{m}$ for the vector whose entries are all equal to 0 except for the $\ell$-th entry, which is equal to 1 .

Definition 4.1. For $m \geq 1$, we define an $m$-structure to be any set of values $\left\{v_{j} \in \mathbb{Q}: \boldsymbol{j} \in \mathbb{N}_{0}^{m}\right\}$ for which there exist $\boldsymbol{c}_{1}, \ldots, \boldsymbol{c}_{d} \in \mathbb{N}_{0}^{m}$ and $\left\{u_{\boldsymbol{j}} \in \mathbb{Z}: \boldsymbol{j} \in \mathbb{N}_{0}^{m} \backslash\{\mathbf{0}\}\right\}$ so that the values satisfy the relation

$$
v_{\boldsymbol{j} \ominus \boldsymbol{c}_{1}}+\cdots+v_{\boldsymbol{j} \ominus \boldsymbol{c}_{d}}=u_{\boldsymbol{j}} \quad \text { for all } \boldsymbol{j} \in \mathbb{N}_{0}^{m} \backslash\{\mathbf{0}\} .
$$

Additionally, we define the following:

1. We say that an $m$-structure is regular if we have that the corresponding vectors $\boldsymbol{c}_{1}, \ldots, \boldsymbol{c}_{d} \in$ $\{0,1\}^{m} \backslash\{\mathbf{0}\}$ for all $1 \leq i \leq d$ as well as $S_{\ell} \neq \emptyset$ for all $1 \leq \ell \leq m$.

2. We say that an $m$-structure is homogeneous outside $\mathbf{t}=\left(t_{1}, \ldots, t_{m}\right) \in \mathbb{N}_{0}^{m}$ if the corresponding vectors $\left\{u_{j} \in \mathbb{Z}: \boldsymbol{j} \in \mathbb{N}_{0}^{m} \backslash\{\mathbf{0}\}\right\}$ satisfy $u_{j}=0$ for all $\boldsymbol{j} \in \mathbb{N}_{0}^{m} \backslash\left[0, t_{1}\right] \times \cdots \times\left[0, t_{m}\right]$.

The first lemma shows a key ingredient in the inductive step developed later by reducing the value of $m$.

Lemma 4.2. For any $m$-structure $\left\{v_{j} \in \mathbb{Q}: \boldsymbol{j} \in \mathbb{N}_{0}^{m}\right\}$ that is homogeneous outside $\boldsymbol{t}=$ $\left(t_{1}, \ldots, t_{m}\right) \in \mathbb{N}_{0}^{m}$ and for which there exists $1 \leq \ell \leq m$ such that $\left|S_{\ell}\right| \neq 0$, the values $\left\{\Delta_{i, \ell}: i \in \mathbb{N}_{0}^{m-1}\right\}$ define an $(m-1)$-structure that is homogeneous outside $\boldsymbol{t}_{\ell}=\left(t_{1}, \ldots, t_{\ell-1}, t_{\ell+1}, \ldots, t_{m}\right)$.

Proof. To simplify notation, assume without loss of generality that $\ell=m$. Let $\mathbf{c}_{1}, \ldots, \mathbf{c}_{d} \in \mathbb{N}_{0}^{m}$ and $\left\{u_{\mathbf{j}} \in \mathbb{Z}: \mathbf{j} \in \mathbb{N}_{0}^{m} \backslash\{\mathbf{0}\}\right\}$ the corresponding sets of vectors given by the definition of $m$-structures.

For $i \in S_{\ell}$, let $\mathbf{c}_{i}^{\prime}=(c(i, 1), \ldots, c(i, m-1))$. Furthermore for $\mathbf{j}^{\prime}=\left(j_{1}, \ldots, j_{m-1}\right) \in \mathbb{N}_{0}^{m-1}$ let $\mathbf{j}=\left(j_{1}, \ldots, j_{m-1}, 0\right)$ and let $u_{\mathbf{j}^{\prime}}=u_{\mathbf{j}+\mathbb{1}_{\ell}}-u_{\mathbf{j}}$. Using this notation, we have

$$
\begin{aligned}
\sum_{i \in S_{\ell}} \Delta_{\mathbf{j}^{\prime} \ominus \mathbf{c}_{i}^{\prime}, \ell} & =\sum_{i \in S_{\ell}} v_{\left(\mathbf{j}+\mathbb{1}_{\ell}\right) \ominus \mathbf{c}_{i}}-\sum_{i \in S_{\ell}} v_{\mathbf{j} \ominus \mathbf{c}_{i}} \\
& =\left(u_{\mathbf{j}+\mathbb{1}_{\ell}}-\sum_{i \in S_{\ell}^{\prime}} v_{\left(\mathbf{j}+\mathbb{1}_{\ell}\right) \ominus \mathbf{c}_{i}}\right)-\left(u_{\mathbf{j}}-\sum_{i \in S_{\ell}^{\prime}} v_{\mathbf{j} \ominus \mathbf{c}_{i}}\right)=u_{\mathbf{j}+\mathbb{1}_{\ell}}-u_{\mathbf{j}}=u_{\mathbf{j}^{\prime}} .
\end{aligned}
$$


Here we have used the fact that for $i \in S_{\ell}^{\prime}$ we have $\left(\mathbf{j}+\mathbb{1}_{\ell}\right) \ominus \mathbf{c}_{i}=\mathbf{j} \ominus \mathbf{c}_{i}$ as $c(i, \ell) \neq 0$. It follows that the values $\left\{\Delta_{\mathbf{i}, \ell}: \mathbf{i} \in \mathbb{N}_{0}^{m-1}\right\}$ form an $(m-1)$-structure with $\left\{\mathbf{c}_{i}^{\prime}: i \in S_{\ell}\right\}$ and $\left\{u_{\mathbf{j}^{\prime}}: \mathbf{j}^{\prime} \in \mathbb{N}_{0}^{m-1} \backslash\{\mathbf{0}\}\right\}$. As $u_{\mathbf{j}^{\prime}}=u_{\mathbf{j}+\mathbb{1}_{\ell}}-u_{\mathbf{j}}=0$ for $\mathbf{j}^{\prime} \in \mathbb{N}_{0}^{m-1} \backslash\left[0, t_{1}\right] \times \cdots \times\left[0, t_{m-1}\right]$, it follows that the structure is homogeneous outside $\mathbf{t}_{\ell}$.

Lemma 4.3. A regular $m$-structure $\left\{v_{j} \in \mathbb{Q}: \boldsymbol{j} \in \mathbb{N}_{0}^{m}\right\}$ that is homogeneous outside $\boldsymbol{t}=$ $\left(t_{1}, \ldots, t_{m}\right) \in \mathbb{N}_{0}^{m}$ satisfies $v_{i}=0$ for all $\boldsymbol{i} \in \mathbb{N}_{0}^{m} \backslash\left[0, t_{1}\right] \times \cdots \times\left[0, t_{m}\right]$.

Proof. We will prove the statement by induction on $m$. Let us start by showing the statement for $m=1$. In this case, $\mathbf{c}_{1}, \ldots, \mathbf{c}_{d}$ are non-zero, positive integers satisfying $\mathbf{c}_{1}=\cdots=\mathbf{c}_{d}=1$ as the structure is regular. It follows that the relations defining the structure are of the type $d v_{\mathbf{j} \ominus 1}=u_{\mathbf{j}}$ for all $\mathbf{j} \in \mathbb{N}$. Since $u_{\mathbf{j}}=0$ for $\mathbf{j}>\mathbf{t}=t_{1}$, we have $v_{\mathbf{i}}=0$ for all $\mathbf{i} \in \mathbb{N}_{0} \backslash\left[0, t_{1} \ominus \mathbf{c}_{1}\right] \subseteq \mathbb{N}_{0} \backslash\left[0, t_{1}\right]$ as desired.

Now assume that the statement is true for all $(m-1)$-structures and let us show that then it must also hold for any $m$-structure. As the structure is regular, we have $S_{\ell} \neq \emptyset$ for all $1 \leq \ell \leq m$ and Lemma4.2 shows that $\left\{\Delta_{\mathbf{i}, \ell}: \mathbf{i} \in \mathbb{N}_{0}^{m-1}\right\}$ is an $(m-1)$-structure that is homogeneous outside $\mathbf{t}_{\ell}$ for any $1 \leq \ell \leq m$. Let us without loss of generality assume that $\ell=m$ to simplify notation. By the inductive assumption it follows that $\Delta_{\mathbf{i}, \ell}=0$ for all $\mathbf{i} \in \mathbb{N}_{0}^{m-1} \backslash\left[0, t_{1}\right] \times \cdots \times\left[0, t_{m-1}\right]$. It follows that $\left\{v_{\mathbf{i}}^{\prime}=v_{\mathbf{i}+\mathbb{1}_{\ell}}: \mathbf{i} \in \mathbb{N}_{0}^{m}\right\}$ is an $m$-structure where the corresponding $\left\{u_{\mathbf{j}}^{\prime}: \mathbf{j} \in \mathbb{N}_{0}^{m}\right\}$ satisfying

$$
u_{\mathbf{j}}^{\prime}= \begin{cases}u_{\mathbf{j}+\mathbb{1}_{\ell}} & \text { for } \mathbf{j}=\left(j_{1}, \ldots, j_{m}\right) \text { s.t. } j_{\ell} \neq 0, \\ u_{\mathbf{j}+\mathbb{1}_{\ell}}+\sum_{i \in S_{\ell}^{\prime}} \Delta_{\mathbf{j} \ominus \mathbf{c}_{i}, \ell} & \text { for } \mathbf{j}=\left(j_{1}, \ldots, j_{m}\right) \text { s.t. } j_{\ell}=0 .\end{cases}
$$

Note that this structure is homogeneous outside $\left(t_{1}, \ldots, t_{m}-1\right)$, that is we have reduced the size of the inhomogeneous part. Repeated application of this principle along all dimensions $1 \leq \ell \leq d$ gives us that

$$
v_{\mathbf{i}}=v_{\mathbf{i}+\mathbb{1}_{\ell}} \text { for all } \mathbf{i} \in \mathbb{N}_{0}^{m} \backslash\left(\left[0, t_{1}\right] \times \cdots \times\left[0, t_{m-1}\right] \times\{0\}\right) \text { and } 1 \leq \ell \leq m .
$$

Considering the relation given by $\mathbf{j}=\left(2 t_{1}, \ldots, 2 t_{m}\right)$, which states that

$$
d v_{\mathbf{j}}=v_{\mathbf{j} \ominus \mathbf{c}_{1}}+\cdots+v_{\mathbf{j} \ominus \mathbf{c}_{d}}=u_{\mathbf{j}}=0 .
$$

Note that the choice of the constant 2 was arbitrary, it just needs to be 'large enough'. It follows that $v_{\mathbf{j}}=0$ and hence, again by relation (10), it follows that $v_{\mathbf{i}}=0$ for all $\mathbf{i} \in \mathbb{N}_{0}^{m} \backslash\left[0, t_{1}\right] \times \cdots \times$ $\left[0, t_{m}\right]$ as desired.

Proof of Theorem 1.1. Recall that $F_{\mathcal{A}}(z)=f_{\mathcal{A}}(z)^{d}$ and that the existence of a set $\mathcal{A}$ for which $r_{\mathcal{A}}\left(n ; k_{1}, \ldots, k_{d}\right)$ is a constant function for $n$ large enough would imply the existence of some polynomial $P(z) \in \mathbb{Z}[z]$ satisfying $P(1) \neq 0$ such that

$$
F_{\mathcal{A}}\left(z^{k_{1}}\right) \cdots F_{\mathcal{A}}\left(z^{k_{d}}\right)=\frac{P^{d}(z)}{(1-z)^{d}} .
$$


Using Proposition 3.1 we see that if a such a function $F_{\mathcal{A}}(z)$ were to exist, then the values $\left\{r_{\mathbf{i}}: \mathbf{i} \in \mathbb{N}_{0}^{m}\right\}$ together with $\mathbf{b}_{1}, \ldots, \mathbf{b}_{m}$ and $\left\{s_{\mathbf{j}}: \mathbf{j} \in \mathbb{N}_{0}^{m} \backslash\{\mathbf{0}\}\right\}$ would define an $m$-structure. By the requirements of the theorem we have $\mathbf{b}_{i} \in\{0,1\}^{m}$ and since $k_{1}, \ldots, k_{d} \geq 2$ we have $\mathbf{b}_{i} \neq \mathbf{0}$. We may also assume that $S_{\ell} \neq \emptyset$ for all $1 \leq \ell \leq d$ as otherwise there exists some $\ell^{\prime}$ such that $q_{\ell^{\prime}} \mid k_{i}$ for all $1 \leq i \leq d$, in which case the representation function clearly cannot become constant, so that this $m$-structure would be regular. It would also be homogeneous outside some appropriate $\mathbf{t} \in \mathbb{N}_{0}^{m}$ as $P(z)$ is a polynomial and hence $s_{\mathbf{j}} \neq 0$ only for finitely many $\mathbf{j} \in \mathbb{N}_{0}^{m}$. Finally, since $r_{\mathbf{i}} \equiv-1 \bmod d$ for all $\mathbf{i} \in \mathbb{N}_{0}^{m}$, this would contradict the statement of Lemma 4.3, proving Theorem 1.1 .

\section{Concluding Remarks}

We have shown that under very general conditions for the coefficients $k_{1}, \ldots, k_{d}$ the representation function $r_{\mathcal{A}}\left(n ; k_{1}, \ldots, k_{d}\right)$ cannot be constant for $n$ sufficiently large. However, there are cases that our method does not cover. This includes those cases where at least one of the $k_{i}$ is equal to 1 . The first case that we are not able to study is the representation function $r_{\mathcal{A}}(n ; 1,1,2)$.

On the other side, let us point out that Moser's construction [3] can be trivially generalized to the case where $k_{i}=k^{i-1}$ for some integer value $k \geq 2$. In view of our results and this construction, we state the following conjecture:

Conjecture 5.1. There exists some infinite set of positive integers $\mathcal{A}$ such that $r_{\mathcal{A}}\left(n ; k_{1}, \ldots, k_{d}\right)$ is constant for $n$ large enough if and only if, up to permutation of the indices, $\left(k_{1}, \ldots, k_{d}\right)=$ $\left(1, k, k^{2}, \ldots, k^{d-1}\right)$, for some $k \geq 2$.

The most likely candidates for a possible counterexample to this conjecture might be those where $\left(k_{1}, k_{2}, k_{3}\right)$ is either $(1,2,6)$ or $(1,2,8)$. One could possibly try to generalise Moser's approach to these scenarios, e.g. by using generalised bases. Understanding these cases would most likely indicate a path towards completely settling the question of Sárközy and Sós.

Acknowledgements. We thank an anonymous referee for comments concerning the complex analytic aspects of our proof. We would also like to thank Oriol Serra for valuable input and fruitful discussions.

\section{References}

[1] J. Cilleruelo and J. Rué. On a question of Sárközy and Sós for bilinear forms. Bulletin of the London Mathematical Society, 41(2):274-280, 2009.

[2] G. Dirac. Note on a problem in additive number theory. Journal of the London Mathematical Society, 1(4):312-313, 1951. 
[3] L. Moser. An application of generating series. Mathematics Magazine, 35(1):37-38, 1962.

[4] J. Rué. On polynomial representation functions for multilinear forms. European Journal of Combinatorics, 34(8):1429-1435, 2011.

[5] A. Sárközy and V. Sós. On additive representation functions. In The mathematics of Paul Erdös I, pages 129-150. Springer, 1997. 